\title{
Looking for new active methods to improve the school performance: Physical activity!
}

\author{
Alberto Ruiz-Ariza ${ }^{1 a}$, Sebastián López-Serrano ${ }^{1}$, Sara Suárez-Manzano ${ }^{1}$, and Emilio J. \\ Martínez-López ${ }^{1}$ \\ ${ }^{1}$ Faculty of Humanities and Education Sciences, University of Jaen, Campus Las Lagunillas s/n, Jaen, \\ Spain
}

\begin{abstract}
The practice of physical activity (PA) has recently been used as a stimulant to improve the cognitive performance in young people and to have positive repercussions on the academic performance [2]. The aims of this research were to conceptualize the relationship between PA and cognition, as well as to associate different physical qualities with better or worse school performance, and thus to be able to make decisions about the kind of PA more adequate to foment from the educative and familiar areas. Our findings are in line with previous literature, and show that a higher physical fitness is associated with better school performance. In conclusion, our study suggests that it is necessary to strengthen the daily PA practice within school context, as well as to raise awareness among families and society about PA promotion.
\end{abstract}

Keywords: Physical activity, families, school performance.

\section{Introduction}

The practice of physical activity (PA) is presented as a great opportunity to improve health, prevent diseases or increase life expectancy [1]. In addition, in recent years, a new neuroscience stream is being focused on studying the effects of PA on the brain and its performance, either short-term or long-term [2]. These findings have reached schools and there are increasing studies that also link PA practice with improvements in academic performance (AP) of children and adolescents [3].

The PA is a very broad term that encompasses several kinds of activities. Among these activities is the physical fitness (PF). The level of PF, especially aerobic fitness, has been associated with better cognition in numerous studies [4]. In the last decade there has been a great increase of studies studying the relation between PF and cognition, however the results are still not conclusive, since few studies analyze the differences in function of the different components of $\mathrm{PF}$, and there are few proposals about these components based on the real educational context $[5,6]$.

\footnotetext{
${ }^{\text {a }}$ Corresponding author: arariza@ujaen.es
} 


\section{Objective}

The present research aims to conceptualize the relationship between PA and cognition, as well as to associate different physical qualities with better or worse school performance, and thus to be able to make decisions about the kind of PA more adequate to foment from the educative and familiar areas.

\section{Sample}

788 adolescents between 12-18 years participated in the present pilot study. The parents, directors and teachers of PE were informed about the study. Informed consent was obtained from parents or legal guardians.

\section{Methodology}

A mixed method was used through bibliographical review, quantitative analysis and theoretical-practical educational proposal.

\subsection{Bibliographic review}

Two specialized databases were used and the search was limited to the last 10 years. The search terms used can be seen in Table 1. The search was conducted in English.

Table 1. Search strategies in databases.

\begin{tabular}{ll}
\hline SportDiscus & TX ("physical fitness" OR fitness OR "physical education") and TX \\
(EBSCO) & ("cognitive performance" OR "academic performance" OR \\
& "academic attainment" OR "academic achievement" OR "academic \\
& outcomes") and TX (adolescent* OR children OR childhood OR \\
& teenager*) \\
Web of & (physical education" OR "physical fitness") AND (cognitive \\
Science & performance" OR "academic performance" OR academic attainment \\
& OR academic achievement OR academic outcomes) \\
& AND (adolescent OR teenager OR children OR childhood) \\
\hline
\end{tabular}

\subsection{Empirical research}

A cross-sectional design was used. The ALPHA-Fitness battery was used [7], and academic qualifications in Mathematics and Language were used for AP [8].

\subsection{Controlled confusion variables}

The socio-educational level was obtained through the educational level of the mother, with the following options: without studies, primary education, secondary education, university education. Mothers were asked directly using a questionnaire [9]. In addition, gender and age were taken into account [8].

\subsection{Statistical analysis}

Data are presented as mean and SD or $\mathrm{n}(\%)$. Differences between genders were compared by one-way ANOVA for continuous variables and the $\chi^{2}$ test for nominal variables. 
Preliminary analyzes showed no significant interaction between variables (all $\mathrm{p}>.05$ ); Therefore, we decided to carry out the analyzes with the total sample. The association of PF and AP was analyzed by linear regression. The regression model was controlled by sex, age, BMI and educational level of the mother. The analyzes were performed with the statistical software SPSS version 22.0 for Windows (SPSS Inc., Chicago). The criterion for statistical significance was set at $\mathrm{p}<.05$.

\section{Results and discussion}

The flow of articles in the search for results can be observed in fig. 1.

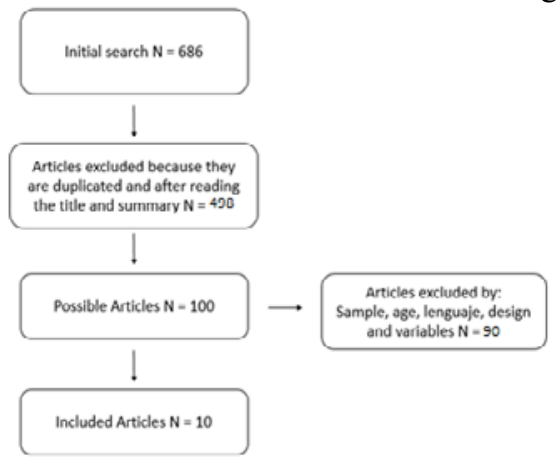

Fig. 1. Flow of articles through the search process.

10 articles were selected. 5 of them were cross-sectional [9-13], 3 interventional [5, 1415], 1 longitudinal, and 1 had a double design (cross-sectional and longitudinal) [16]. PF variables were aerobic capacity, muscle strength, motor ability, coordination and flexibility. Over sixty percent of the studies included covariates, including socio-educational level of families, BMI, sex, and age. Most of studies showed a positive relationship between PF and cognition. Aerobic capacity, motor ability, coordination and perceptual ability are the components that are mostly associated with cognition in young people, however, the relationship to muscle strength or flexibility is unclear.

On the other hand, our own empirical cross-sectional research, has also shown that PF is associated with AP after adjusting by sex, age, BMI and maternal education. Specify, aerobic and motor ability were associated with Mathematic and Spanish Language. However, muscular strength is no related with any AP variable.

Previous studies have shown that it is related to better performance in several cognitive abilities, such as inhibitory control, working memory and attention [7]. In this sense, proposing intervention programs that include aerobic and motor training may improve PF, and therefore have positive effects on the brain. Some explanations may be the neurogenesis, angiogenesis or synaptogenesis that could increase the brain activity and the number of neuronal synapses, leading to increased BDNF and the reorganization of motor representations of motor cortex [17], with direct effect on cognitive performance and academic achievement.

\section{Educational proposal}

Based on our findings and the studies of [5, 6], and [3], we intend to include the practice of PA as a tool to increase cognition in the Educational Center. To this one, we propose: 
- In PE classes: To create didactic units that have as objective the increase of the aerobic capacity. For example by including the HIIT method [18]. In addition, include the training of motor skill and coordination through agility circuits and the use of implements.

- Just before each class: 10 minutes of coordination exercise and motor skill improves attention and concentration, so we recommend that you spend that time before each class to activities of this type [14]. This would lead to an approximate daily increase of 60 minutes of PA practice (minimum recommended by WHO).

-Between classes: Include breaks of 5-8 minutes in the middle of the classes to make some coordination or motor skill play with the aim of arousing attention and concentration, for example: The ten passes with the non-dominant hand with a tennis ball, and having than keeping a leg still as a pivot.

- Just before and after the school day: Encourage active commuting to and from the Educational Center. Previous studies have already demonstrated its benefits at the cognitive level [19].

- Extracurricular PA: The practice of aerobic or motor PA during out-of-school hours may also be beneficial for the cognitive performance of young people [9], so fostering this practice from the public and family sectors is crucial.

\section{Conclusion}

In conclusion, aerobic capacity, motor ability and coordination may have a potential influence on the cognition of adolescents. We suggest that PE teachers give more importance to the aerobic and motor component in their classes and we encourage the promotion of joint actions in a multidisciplinary way between PE subjects and the instrumental subjects, as well as to promote an increase in aerobic fitness and motor skills during adolescence, from the educational, political and family spheres.

\section{References}

1. World Health Organisation. Extracted on 02 march 2017 in http://www.who.int/topics/physical_activity/es/ (2015)

2. A. Ruiz-Ariza, A. Grao-Cruces, N.E.M. de Loureiro, E.J. Martínez-López, International Review of Sport and Exercise Psychology, 1, 108 (2017)

3. M.J. Mullender-Wijnsma, E. Hartman, J.W. de Greeff, R.J. Bosker, S. Doolaard, C, Visscher, Journal of School Health, 6, 365 (2015)

4. M.A.I. Aberg, N.L. Pedersen, K. Torén, M. Svartengren, B. Bäckstrand, T. Johnsson, ... H.G. Kuhn, Proceedings of the National Academy of Sciences of the United States of America, 49, 20906 (2009)

5. D.N. Ardoy, J.M. Fernández-Rodríguez, D. Jiménez-Pavón, R. Castillo, J.R. Ruiz, F.B. Ortega, Scandinavian Journal of Medicine \& Science in Sports, 1, 52 (2014)

6. J.E. Donnelly, K. Lambourne, Preventive Medicine, 52, 36 (2011)

7. J.R. Ruiz, J. Castro-Pinero, V. Espana-Romero, E.G. Artero, F.B. Ortega, M.M. Cuenca, ...M.J. Castillo, British Journal of Sports Medicine, 6, 518 (2011)

8. A. Ruiz-Ariza, J. Ruiz, M. De la Torre-Cruz, P. Latorre-Román, E.J. Martínez-López, Revista Latinoamericana de Psicologia, in press, (2015)

9. I. Esteban-Cornejo, C.M. Tejero-González, D. Martinez-Gomez, J. del-Campo, A. González-Galo, C. Padilla-Moledo, ...O.L. Veiga, The Journal of Pediatrics, 2, 306 (2014)

10. R.W. Bass, D.D. Brown, K.R. Laurson, M.M. Coleman, Acta Paediatrica, 8, 832 (2013) 
11. D.P. Coe, T. Peterson, C. Blair, M.C. Schutten, H. Peddie, The Journal of School Health, 7, 500 (2013)

12. J.C. Janak, K.P Gabriel., A.O. Oluyomi, A. Peréz, H.W. Kohl, S H. Kelder, The Journal of School Health, 8, 533 (2014)

13. L.B. Sardinha, A. Marques, S. Martins, A. Palmeira, C. Minderico, BMC Pediatrics, 1, $176(2014)$

14. H. Budde, C. Voelcker-Rehage, S. Pietraßyk-Kendziorra, P. Ribeiro, G. Tidow, Neuroscience Letters, 2, 219 (2008)

15. A.K. Travlos, International Journal of Sport and Exercise Psychology, 3, 302 (2010)

16. L.J. Chen, K.R. Fox, P.W. Ku, C.Y. Taun, The Journal of School Health, 9, 631 (2013)

17. D.L. Adkins, J. Boychuk, M.S. Remple, J.A. Kleim, Journal of Applied Physiology, 6, 1776 (2006)

18. S.A. Costigan, N. Eather, R.C. Plotnikoff, D.R. Taaffe, D.R. Lubans, British Journal of Sports Medicine, 19, 1253 (2015)

19. D. Martínez-Gómez, J.R. Ruiz, S. Gómez-Martínez, P. Chillón, J.P. Rey-López, L.E. Díaz, ...A. Marcos, Archives of Pediatrics \& Adolescent Medicine, 4, 300 (2011) 